\title{
Enhanced Two-Dimensional Data-aided Channel Estimation for TDS-OFDM
}

\author{
Ming Liu, Matthieu Crussière, Jean-François Hélard \\ European University of Brittany (UEB), INSA-Rennes, \\ Institute of Electronics and Telecommunications of Rennes (IETR), France \\ Email: \{first name. last name $\} @$ insa-rennes.fr
}

\begin{abstract}
In time domain synchronous (TDS)-OFDM, the channel estimation is conventionally carried out based on the pseudo noise (PN) sequence. The PN sequence based channel estimation however suffers interference from adjacent OFDM data symbols. This paper proposes a new low-complexity dataaided channel estimation method with two-dimensional (2-D) estimate refinement and interpolation. Data-aided channel estimation is carried out using the rebuilt OFDM data symbols as virtual training symbols. In contrast to the classical turbo channel estimation, interleaving and decoding functions are not used when rebuilding OFDM data symbols thereby reducing the complexity. 2-D estimate refinement and interpolation are proposed to improve the data-aided channel estimation. Simulation results show that the performance of TDS-OFDM based DTMB system using the proposed method is very close to that with perfect channel estimation in terms of bit error rate (BER).
\end{abstract}

\section{INTRODUCTION}

Orthogonal frequency division multiplexing (OFDM) attracts much attention for high data rate communications due to its merits of multipath immunity and bandwidth efficiency. Traditionally, cyclic prefix (CP) is inserted as guard interval (GI) between two adjacent OFDM symbols to mitigate intersymbol interference (ISI) and enable simple equalization at receiver side. Recently, an alternative type of GI which is composed of a known pseudo noise (PN) sequence is proposed for the purpose of improved spectrum efficiency and fast tracking. This new OFDM signal waveform is referred to as time domain synchronous (TDS)-OFDM ${ }^{1}$ [1]. TDS-OFDM has been adopted by the Chinese digital terrestrial multimedia broadcasting (DTMB) system [2].

In TDS-OFDM, the PN sequence in GI is reused as training sequence for channel estimation. Since the PN sequence based channel estimation is affected by the adjacent OFDM data symbols [3], several approaches were proposed to remove the influence of data symbol from PN sequence [1] [3] [5]. However, the performance is degraded either by ISI from previous data symbol in [1] or by the error of interference estimation in [3] or by error propagation resulting from the hard decision of data symbols in [5]. A better solution is the iterative turbo channel estimation [6] which rebuilds the data symbols using the decoded information bits and then uses the rebuilt data symbols as known training symbols for channel estimation. The computational complexity and time

\footnotetext{
${ }^{1}$ It is also termed as known symbol padding (KSP)-OFDM [3] and pseudo random postfix (PRP)-OFDM [4].
}

delay of this method are however very high especially for systems with sophisticated decoder and deep interleaver. For example, the DTMB system includes a low density parity check (LDPC) encoder followed by an interleaver of depth 170 (or 510) OFDM symbols, which makes the turbo channel estimation impracticable for real use. [7] proposed a dataaided channel estimation method that excludes the channel decoding and interleaving from the feedback loop to reduce the computational complexity. However, the use of this method is limited to uniform-power modulations e.g. BPSK and QPSK.

In this paper, we propose a new two-dimensional (2-D) channel estimation method which not only enables the use with non-uniform-power (higher order) modulations but, more importantly, improves the channel estimation performance with reduced complexity. More concretely, refined channel estimates are first obtained for some 'virtual pilot' positions located in the time-frequency plan by averaging the instantaneous data-aided channel estimates within the coherence region. 2-D Wiener filtering based interpolation is then performed to get an overall data-aided channel estimation. Several virtual pilot patterns are also studied in this paper. Simulation results show that the proposed method can provide nearoptimal performance.

The rest of the paper is organized as follows. In section II, the mobile channel and TDS-OFDM signal models are described. In section III, the proposed 2-D data-aided channel estimation method is presented. Simulation results are shown in section IV. Conclusions are drawn in section V.

In this paper, $(\cdot)^{*}$ denotes the conjugate of complex number, $\mathbb{E}\{\cdot\}$ is the expected value, $(\cdot)^{T}$ and $(\cdot)^{H}$ are the matrix transpose and Hermitian transpose, respectively.

\section{System MOdEL}

\section{A. Channel Model}

The time-variant channel impulse response (CIR) with $L$ distinct paths is described as:

$$
h(\tau, t)=\sum_{l=0}^{L-1} \gamma_{l}(t) \delta\left(\tau-\tau_{l}\right)
$$

where $\tau_{l}$ and $\gamma_{l}(t)$ are the delay and the gain of the $l^{t h}$ path, respectively. $\gamma_{l}(t)$ is assumed to be zero mean complex Gaussian process which is mutually independent for different paths. In this paper, the channel is assumed to be quasi-static, 


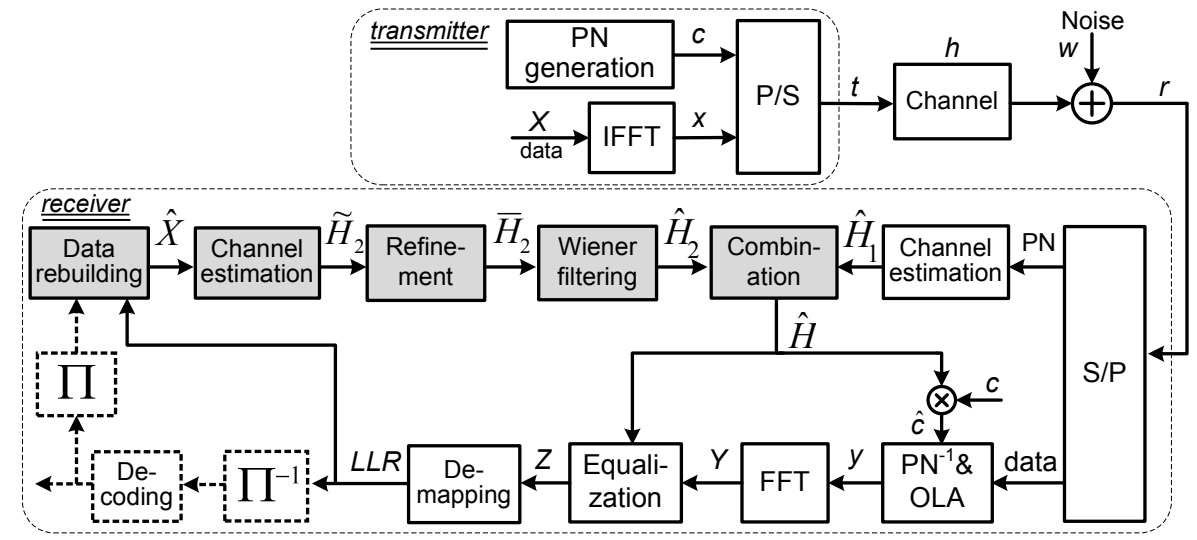

Fig. 1. Block diagram of TDS-OFDM system. The shaded blocks are the processings used for the proposed method. The dashed blocks are the additional processings needed for the turbo channel estimation.

namely constant within one OFDM symbol but changing from one symbol to another. The channel frequency response (CFR) for the $k^{t h}$ subcarrier of the $i^{t h}$ OFDM symbol is:

$$
H[i, k] \triangleq H(i T, k \Delta f)=\sum_{l=0}^{L-1} \gamma_{l}(i T) e^{-j 2 \pi k \Delta f \tau_{l}},
$$

where $H(t, f)$ is the Fourier transform of the CIR, $T$ is the time duration of one OFDM symbol and $\Delta f=\frac{1}{T}$ is the subcarrier spacing. Assuming the channel has the same correlation function for all paths, the time-frequency correlation function of the CFR can be represented by the product of time domain and frequency domain correlations [8]:

$$
\phi_{H}[p, q] \triangleq \mathbb{E}\left\{H[i+p, k+q] H^{*}[i, k]\right\}=\sigma_{h}^{2} r_{t}[p] r_{f}[q],
$$

where $\sigma_{h}^{2}$ is the total average power of CIR. Without loss of generality, we assume $\sigma_{h}^{2}=1 . r_{t}[p]$ and $r_{f}[q]$ are the time and frequency domain correlation functions of the CFR, respectively. Concretely, taking the Jakes' mobile channel model, $r_{t}[p]$ is:

$$
r_{t}[p]=J_{0}\left(2 \pi p f_{d} T\right),
$$

where $J_{0}(\cdot)$ is the zeroth-order Bessel function of the first kind, and $f_{d}$ is the Doppler frequency related to velocity $v$ and carrier frequency $f_{c}$ by $f_{d}=v f_{c} / c$, where $c$ is the speed of light. The frequency domain correlation $r_{f}[q]$ is:

$$
r_{f}[q]=\sum_{l=0}^{L-1} \sigma_{l}^{2} e^{-j 2 \pi q \Delta f \tau_{l}} .
$$

where $\sigma_{l}^{2}$ is the variance of the $i^{t h}$ path amplitude.

\section{B. Baseband TDS-OFDM Signal Model}

Fig.1 presents the baseband model of the TDS-OFDM. The $i^{\text {th }}$ OFDM symbol is formed by $N$-point inverse fast Fourier transform (IFFT):

$$
x(i, n)=\frac{1}{\sqrt{N}} \sum_{k=0}^{N-1} X[i, k] e^{j \frac{2 \pi}{N} n k}, 0 \leq n \leq N-1 .
$$

A $\nu$-length PN sequence $\{c(i, n)\}_{n=-\nu}^{-1}$ is then inserted before $\{x(i, n)\}_{n=0}^{N-1}$ as GI. The $i^{t h}$ transmitted time domain signal is thus:

$$
t(i, n)=\left\{\begin{array}{cc}
c(i, n) & -\nu \leq n<0 \\
x(i, n) & 0 \leq n \leq N-1
\end{array}\right.
$$

After passing the multipath fading channel, the received signal is a linear convolution of the transmitted signal and the CIR. After PN sequence removal and overlap and add (OLA) process, the linear convolution becomes a circular one [7]. The received frequency domain TDS-OFDM signal $Y$ has finally a similar representation as the CP-OFDM:

$$
Y[i, k]=H[i, k] X[i, k]+W^{\prime}[i, k]
$$

where $W^{\prime}$ is the equivalent noise component which is slightly colored after OLA process with an equivalent variance of $\sigma_{W^{\prime}}^{2}=\frac{N+\nu}{N} \sigma_{w}^{2}$ given $\sigma_{w}^{2}$ the variance of the additive white Gaussian noise [3]. The TDS-OFDM signal can thus be easily equalized by a one-tap equalizer.

\section{PN based Channel Estimation}

As specified in [2], the $\nu$-length PN sequence in the GI is composed of a $N_{P N}$-length PN sequence and its CP. If this CP is longer than the length of CIR, the $N_{P N}$-length PN sequence is ISI free. Using the ISI free PN sequence, a least square (LS) channel estimation is made for the $i^{\text {th }}$ OFDM symbol:

$$
\bar{H}_{1}[i, k]=\frac{S[i, k]}{P[i, k]}=H[i, k]+\frac{W[i, k]}{P[i, k]}, 0 \leq k<N_{P N},
$$

where $P$ and $S$ are obtained through $N_{P N}$-length fast Fourier transform (FFT) applied on the transmitted and received ISI free PN sequences, respectively. The $N$-length CFR estimation $\hat{H}_{1}$ is obtained by FFT-based interpolation. The mean square error of $\hat{H}_{1}$ is:

$$
\varepsilon_{\hat{H}_{1}}=\frac{1}{N} \sum_{k=0}^{N-1} \mathbb{E}\left\{\left|H[i, k]-\hat{H}_{1}[i, k]\right|^{2}\right\}=\frac{L \sigma_{w}^{2}}{N_{P N}} \sum_{k=0}^{N_{P N}-1} \frac{1}{|P[i, k]|^{2}} .
$$




\section{Proposed 2-D DATA-AIDED ChANNEL Estimation METHOD}

The proposed 2-D data-aided channel estimation method consists of three steps. First, a PN sequence based channel estimation as described above is carried out. The estimated CIR is used to remove the PN from the received TDS-OFDM signal and then make the equalization. Secondly, the 2-D dataaided channel estimation is made to provide a new channel estimate. Finally, the two channel estimates are combined using the minimum mean squared error (MMSE) criteria.

\section{A. Proposed Data-aided Channel Estimation}

In contrast to a classical iterative turbo channel estimation algorithm [6], the proposed method excludes the deinterleaving, channel decoding and interleaving processings from the feedback loop as shown in Fig. 1. In other words, the estimated data symbols used for data-aided channel estimation are rebuilt directly using the output information of the demapper.

The soft-output demapper demodulates the complex data symbols into Log-likelihood ratio (LLR) of bits [9]. The LLR $\lambda_{l}[i, k]$ corresponding to the $l^{t h}$ bit of the $(i, k) t h$ equalized data symbol $Z[i, k]$ is defined as:

$$
\lambda_{l}[i, k] \triangleq \log \frac{P(b[i, k, l]=1 \mid Z[i, k])}{P(b[i, k, l]=0 \mid Z[i, k])},
$$

where the $P(b[i, k, l]=1 \mid Z[i, k])$ is the conditional probability of the $l^{t h}$ bit equal to 1 given $Z[i, k]$. The sign of the LLR decides the corresponding bit equal to 1 or 0 , and its absolute value gives the reliability of the decision. Based on the LLR, the probabilities of a bit equal to 1 and 0 are $P(b[i, k, l]=1)=\frac{e^{\lambda_{l}(k)}}{1+e^{\lambda_{l}(k)}}$ and $P(b[i, k, l]=0)=$ $1-P(b[i, k, l]=1)$, respectively. They are then used as $a$ priori probabilities to estimate the data symbol. First, the probability that the transmitted symbol $X[i, k]$ is equal to a specific constellation point $\alpha_{j}$ is computed as the product of the probabilities of all the bits belonging to this constellation:

$$
P\left(X[i, k]=\alpha_{j}\right)=\prod_{l=1}^{\log _{2} \mu} P\left(b[i, k, l]=\kappa_{l}\left(\alpha_{j}\right)\right),
$$

where $\Psi$ is the set of the constellation points of a given modulation scheme, $\mu$ is the modulation order and $\kappa_{l}\left(\alpha_{j}\right) \in\{0,1\}$ is the value of the $l^{t h}$ bit of the constellation point $\alpha_{j}$. The soft data symbol is an expected value taking the a priori probabilities (12) into account:

$$
\hat{X}[i, k]=\sum_{\alpha_{j} \in \Psi} \alpha_{j} \cdot P\left(X[i, k]=\alpha_{j}\right) .
$$

Note that, being different from the classical decision feedback channel estimation methods [5], [8], no decision is made here in order to prevent error propagation. Directly using the soft data symbols, an instantaneous channel estimate is obtained over all active subcarriers by:

$$
\begin{gathered}
\tilde{H}_{2}[i, k]=\frac{1}{E_{\hat{X}}[i, k]} \hat{X}[i, k]^{*} Y[i, k] \\
=\frac{\hat{X}[i, k]^{*} X[i, k]}{E_{\hat{X}}[i, k]} H[i, k]+\frac{1}{E_{\hat{X}}[i, k]} \hat{X}[i, k]^{*} W^{\prime}[i, k],(14)
\end{gathered}
$$

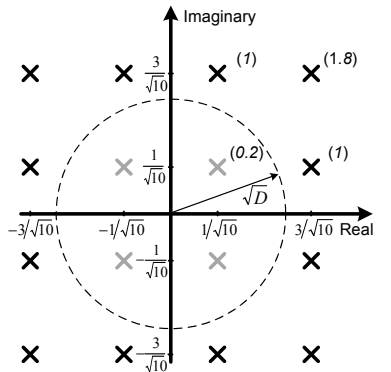

(a) 16QAM.

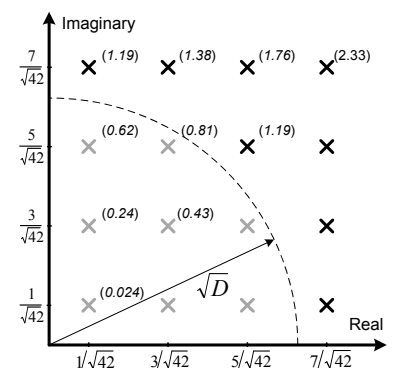

(b) 64QAM (first quadrant).
Fig. 2. Select the constellation points with high power (marked black). The values in the bracket are the normalized power of the constellation points.

where $E_{\hat{X}}[i, k]=|\hat{X}[i, k]|^{2}$ is the power of the $(i, k)$ th soft data symbol which is used as power normalization factor. In contrast to the uniform-power modulation cases e.g. BPSK and QPSK where the normalization factor is an accurately known value [7], the normalization factor should be estimated in the non-uniform-power modulation cases e.g. 16QAM and 64QAM. If the data symbols are perfectly rebuilt (e.g. signalto-noise ratio is high), namely $\hat{X}[i, k]=X[i, k]$, (14) turns to an LS estimator:

$$
\tilde{H}_{2}[i, k]=H[i, k]+\frac{W^{\prime}[i, k]}{\sqrt{E_{X}[i, k]}} .
$$

Since the rebuilt soft data symbols $\hat{X}$ are affected by the noise as well as the channel fading, the instantaneous CFR estimates (14) are inaccurate for some subcarriers. (15) indicates that the estimation error is reciprocally proportional to the power of the data symbol used to make the estimation. It means that the CFR estimates obtained using the data symbols with higher power are more reliable. Therefore, in order to achieve better estimation results, we propose to set a power threshold $D$ to select data symbols with higher power for channel estimation as shown in Fig.2. Note that the threshold is used to select the reliable data symbols but not to make hard decision to the data symbols. The side effect of this selection is that the CFR estimates are not available for all subcarriers and the available CFR estimates locate on random positions.

It is known that given sufficient time-frequency samples, the CFR can be rebuilt using some interpolation techniques, e.g. linear, FFT-based, MMSE interpolations. Among all methods, the MMSE interpolation based on Wiener filtering provides the best mean squared error (MSE) performance [11]. However, the randomly located CFR estimates require to compute the coefficients of the Wiener filter by means of matrix inversion for each OFDM symbol. The computational complexity is unaffordable when the FFT size is large. Fortunately, as both the time delay spread and Doppler spectrum of the channel are limited, the CFR is highly correlated, i.e. almost identical within the coherence bandwidth and coherence time [13]. Moreover, the coherence bandwidth spreads over several adjacent subcarriers in the OFDM system with large FFT size, and the coherence time is normally longer than several OFDM symbol durations in low and medium velocity cases. 


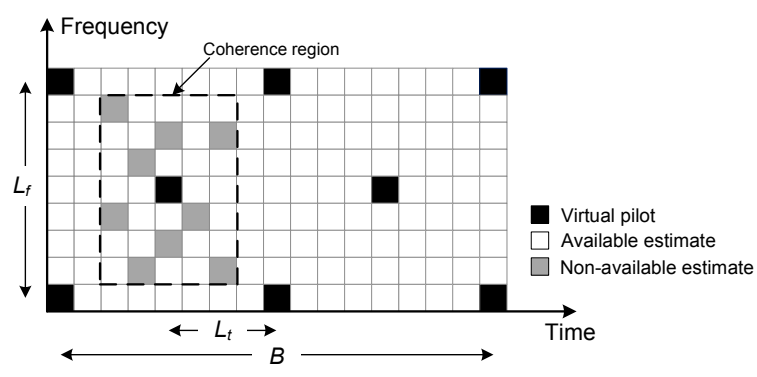

Fig. 3. 2-D average in time and frequency, with diamond shape pilot pattern.

This enables us to carry out a 2-D estimate refinement and interpolation to provide a better channel estimation.

\section{B. 2-D CFR Estimate Refinement}

The 2-D estimate refinement and interpolation is carried out over a number of OFDM symbols within an interpolation block as shown in Fig. 3. The block size $B$, i.e. the number of OFDM symbols in the block, is chosen according to the latency, storage and complexity considerations. Suppose that the set of indices of available CFR estimates in an interpolation block is $\boldsymbol{\Theta}$. For low-complexity consideration, some indices $\left(i_{p}, k_{p}\right) \in \boldsymbol{\Xi}$ are pre-selected as 'virtual pilots', where $\boldsymbol{\Xi}$ is the set of time-frequency indices of virtual pilots. Then, a refined CFR estimate for a virtual pilot is computed by averaging all the available CFR estimates within the coherence region bounded by the coherence bandwidth and time, as shown in Fig. 3. The size of the coherence region is chosen less than or equal to the coherence bandwidth and time. It can be either empirically pre-selected according to the worst case, or adaptively chosen by using the information obtained in the initial PN based channel estimation. The refined CFR estimate for the pilot located at $\left(i_{p}, k_{p}\right)$ is:

$$
\begin{aligned}
& \bar{H}_{2}\left[i_{p}, k_{p}\right]=\frac{1}{M} \sum_{p, q \in \Theta_{i_{p}, k_{p}}} \tilde{H}_{2}[p, q] \\
= & \frac{H\left[i_{p}, k_{p}\right]}{M} \sum_{p, q \in \Theta_{i_{p}, k_{p}}} \frac{\hat{X}[p, q]^{*} X[p, q]}{E_{\hat{X}}[p, q]}+\frac{W^{\prime}}{M_{p, q \in \Theta_{i_{p}, k_{p}}}} \frac{\hat{X}[p, q]^{*}}{E_{\hat{X}}[p, q]} \\
\simeq & H\left[i_{p}, k_{p}\right]+\frac{W^{\prime}}{M} \sum_{p, q \in \Theta_{i_{p}, k_{p}}} \frac{\hat{X}[p, q]^{*}}{E_{\hat{X}}[p, q]}
\end{aligned}
$$

where $\Theta_{i_{p}, k_{p}} \in \boldsymbol{\Theta}$ is the subset of available CFR estimates within the coherence region with $\left(i_{p}, k_{p}\right)$ its central position and $M$ is the cardinality of $\Theta_{i_{p}, k_{p}}$. The variance of the estimation error in (16) is then:

$$
\begin{aligned}
& \sigma_{\bar{H}_{2}}^{2}[i, k]=\mathbb{E}\left\{\left|H[i, k]-\bar{H}_{2}[i, k]\right|^{2}\right\} \\
= & \mathbb{E}\left\{\left|\frac{W^{\prime}}{M} \sum_{p, q} \frac{\hat{X}[p, q]}{E_{\hat{X}}[i, k]}\right|^{2}\right\}=\frac{\sigma_{W^{\prime}}^{2}}{M^{2}} \sum_{p, q}\left|\frac{\hat{X}[p, q]}{E_{\hat{X}}[p, q]}\right|^{2} \\
= & \frac{\sigma_{W^{\prime}}^{2}}{M^{2}} \sum_{p, q} \frac{1}{E_{\hat{X}}[p, q]}
\end{aligned}
$$

Particularly, if only the data symbols with power higher than 1, i.e. $E_{\hat{X}}[p, q] \geq 1$, are used for estimation, the estimation error is bounded by $\sigma_{\bar{H}_{2}}^{2} \leq \sigma_{W^{\prime}}^{2}$.

By repeating this process over all virtual pilots in the interpolation block, a number of refined CFR estimates are obtained. With these reliable estimates, the overall CFR estimate can thus be obtained via interpolation.

\section{2-D Wiener Filtering based Interpolation}

The 2-D Wiener filtering outperforms other interpolation techniques, e.g. linear interpolation, FFT-based interpolation etc. However its computational complexity is very high. The use of two concatenated 1-D Wiener filters, i.e. one in frequency domain and then the other in time domain, significantly reduces the computational complexity with negligible performance degradation compared to the optimum 2-D Wiener filter [10]. The $2 \times 1-\mathrm{D}$ Wiener filtering is represented as:

$$
\hat{H}_{2}[i, k]=\underbrace{\sum_{i_{p}} \omega_{t}\left[i, k, i_{p}\right]}_{\text {time domain }} \underbrace{\sum_{k_{p}} \omega_{f}\left[k, i_{p}, k_{p}\right]}_{\text {frequency domain }} \bar{H}_{2}\left[i_{p}, k_{p}\right] \text {, }
$$

where $\omega_{t}$ 's and $\omega_{f}$ 's are the coefficients of the 1-D Wiener filter in time and frequency domains, respectively, while $K_{f}=\left\lfloor\frac{N}{L_{f}}\right\rfloor$ and $K_{t}=\left\lfloor\frac{B}{L_{t}}\right\rfloor$ are the number of virtual pilots within one OFDM symbol and the number of OFDM symbols including virtual pilots, respectively. $L_{f}$ and $L_{t}$ are the pilot spacings in frequency and time domains. The coefficients of frequency domain Wiener filter can be computed as:

$$
\boldsymbol{\omega}_{f}=\boldsymbol{\Phi}_{f}^{-1} \boldsymbol{\theta}_{f}
$$

where $\boldsymbol{\Phi}_{f}=\mathbb{E}\left\{\overline{\mathbf{H}}_{2} \overline{\mathbf{H}}_{2}^{H}\right\}=\left(\mathbf{R}_{f}^{(1)}+\sigma_{\bar{H}_{2}}^{2} \mathbf{I}\right)$ is the $K_{f} \times K_{f}$ autocorrelation matrix of CFR estimates on pilot positions obtained in (16), I is identity matrix. $\boldsymbol{\theta}_{f}=\mathbb{E}\left\{\overline{\mathbf{H}}_{2} \mathbf{H}\right\}=\mathbf{R}_{f}^{(2)}$ is the $K_{f} \times N$ cross-correlation matrix of CFR estimates $\overline{\mathbf{H}}_{2}$ and the real CFR $\mathbf{H} . \mathbf{R}_{f}^{(1)}$ and $\mathbf{R}_{f}^{(2)}$ are the autocorrelation matrix of the real CFR in frequency domain, and its components can be computed by (5). The MSE of the CFR estimate after frequency domain filtering is:

$$
\varepsilon_{f}=\frac{1}{N} \operatorname{Tr}\left(\mathbf{R}_{f}^{(3)}-\mathbf{R}_{f}^{(2) T} \boldsymbol{\Phi}_{f}^{-1} \mathbf{R}_{f}^{(2) *}\right),
$$

where $\operatorname{Tr}(\cdot)$ is the trace operation, $\mathbf{R}_{f}^{(3)}$ is the $N \times N$ autocorrelation matrix of CFR. Similarly, the coefficients of the time domain Wiener filter can be computed as:

$$
\boldsymbol{\omega}_{t}=\boldsymbol{\Phi}_{t}^{-1} \boldsymbol{\theta}_{t}
$$

where $\boldsymbol{\Phi}_{t}$ and $\boldsymbol{\theta}_{t}$ have the same definition as $\boldsymbol{\Phi}_{f}$ and $\boldsymbol{\theta}_{f}$ except that the components of autocorrelation matrix of CFR are computed according to (4). The MSE of the CFR estimate after frequency domain filtering is:

$$
\varepsilon_{\hat{H}_{2}}=\frac{1}{B} \operatorname{Tr}\left(\mathbf{R}_{t}^{(3)}-\mathbf{R}_{t}^{(2) T} \boldsymbol{\Phi}_{t}^{-1} \mathbf{R}_{t}^{(2) *}\right) .
$$

Since the positions of the virtual pilots are pre-selected, the matrix inversion $\boldsymbol{\Phi}_{f}^{-1}$ and $\boldsymbol{\Phi}_{t}^{-1}$ in the filter coefficients computation can be pre-computed and used for all OFDM symbols to reduce the computational complexity. 


\section{MMSE Combination}

When the PN based and data-aided channel estimates are obtained, a linear combination is proposed to get an improved CFR estimate:

$$
\hat{H}=\beta \hat{H}_{1}+(1-\beta) \hat{H}_{2} .
$$

For the DTMB system, since all subcarriers are active, this combination is carried out over all subcarriers. Otherwise, the combination is only made for the active subcarriers and the CFR estimates for null subcarriers are kept as the results obtained from the PN based one. The optimum weight values $\beta_{\text {opt }}$ can be obtained using the MMSE criteria:

$$
\begin{array}{r}
\beta_{\text {opt }}=\arg \min _{\beta}\left\{E\left\{|H-\hat{H}|^{2}\right\}\right\} \\
=\arg \min _{\beta}\left\{\beta^{2} \varepsilon_{\hat{H}_{1}}+(1-\beta)^{2} \varepsilon_{\hat{H}_{2}}\right\} .
\end{array}
$$

The above equation uses the fact that $\hat{H}_{1}$ and $\hat{H}_{2}$ are obtained from different sources and thus uncorrelated. Since the MSE function is convex, the $\beta_{o p t}$ is obtained by setting the derivative with respect to $\beta$ equal to zero and the solution is:

$$
\beta_{o p t}=\frac{\varepsilon_{\hat{H}_{2}}}{\varepsilon_{\hat{H}_{1}}+\varepsilon_{\hat{H}_{2}}} .
$$

After a given maximum number of iterations, the combined CFR (23) estimate is output as the final CFR estimate. Otherwise it is used as the initial channel estimate in the next iteration for PN subtraction and equalization.

\section{E. Complexity Analysis}

The additional computational complexity of the proposed channel estimation method is evaluated in terms of required real multiplications and real additions for each OFDM symbol. The data rebuilding process including (11), (12) and (13) needs $\left(N \cdot \mu \cdot \log _{2} \mu+N \cdot 2 \log _{2} \mu\right)$ multiplications and $N \cdot 2 \log _{2} \mu$ additions. The instantaneous data-aided estimation (14) needs $6 N$ multiplications and $2 N$ additions. The averaging in coherence region (16) requires $2 N$ additions and a few times of division which are neglected. The frequency domain Wiener filtering needs $2 N \cdot \frac{K_{f}}{L_{t}}$ additions and multiplications, while the time domain Wiener filtering requires $2 N \cdot K_{t}$ additions and multiplications. The MMSE combination needs $4 N$ multiplications and $2 N$ additions. In all, $N \cdot\left((\mu+2) \log _{2} \mu+10+2 \frac{K_{f}}{L_{t}}+2 K_{t}\right)$ multiplications and $N \cdot\left(2 \log _{2} \mu+6+2 \frac{K_{f}}{L t}+2 K_{t}\right)$ additions are needed, which is between $\mathcal{O}(N)$ and $\mathcal{O}\left(N^{2}\right)$. Interestingly, the proposed blocktype 2-D channel estimation method has a complexity less than the symbol-by-symbol 1-D processing manner [7] which needs $N \cdot\left((\mu+2) \log _{2} \mu+14+2 K_{f}\right)+2 K_{f}$ multiplications and $N \cdot\left(2 \log _{2} \mu+8+2 K_{f}\right)$ additions. Even lower complexity can be achieved by reducing the size of Wiener filter as in [11].

\section{Simulation Results}

Simulation parameters are chosen from the DTMB system [2]. The length of the GI is set to $\nu=420$. Both the convolutional interleaver and LDPC code are included in the

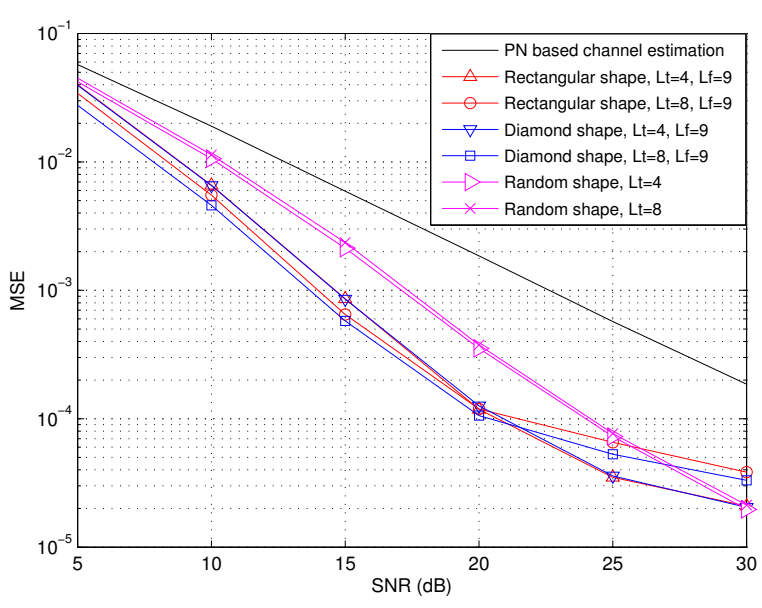

Fig. 4. MSE of the proposed data-aided channel estimation for 16QAM after 2 iterations in TU-6 channel with velocity of $30 \mathrm{~km} / \mathrm{h}$.

simulation in order to perform a system level evaluation. The COST207 Typical Urban (TU-6) channel model is employed in the simulation. The coherence bandwidth of TU-6 channel is $18.8 \mathrm{kHz}$ equivalent to 9 subcarrier spacings. The mobile speed is set to $30 \mathrm{~km} / \mathrm{h}$ and $120 \mathrm{~km} / \mathrm{h}$ which correspond to maximum Doppler frequency $13.89 \mathrm{~Hz}$ and $55.56 \mathrm{~Hz}$, respectively, given the carrier frequency $500 \mathrm{MHz}$. The coherence time is $0.0305 \mathrm{~s}$ and $0.0076 \mathrm{~s}$ equivalent to 61 and 15 OFDM symbols, respectively. Without loss of generality, the size of the coherence region is set equal to the pilot spacing. The data symbol selection threshold $D$ is chosen to be 0.9 for 16QAM and 0.75 for 64QAM in the simulation, respectively.

Fig.4 presents the MSE performance of proposed method adopting rectangular shape, diamond shape and random shape pilot patterns. All the three cases use the same time domain Wiener filter. The difference lies in the pilot arrangement in frequency domain. In the rectangular shape case, the pilot position is fixed with a spacing of $L_{f}$ subcarriers for all OFDM symbols that carry virtual pilots, while in the diamond shape case, the pilot position circularly shifts $\frac{L_{f}}{2}$ from one OFDM symbol to the next as shown in Fig.3. Note that the latter is known as the optimum pilot pattern for channel estimation [12]. As for the random pilot shape case, all available instantaneous CFR estimates are fed to the frequency domain 1-D Wiener filter to compute the CFR over all subcarriers, and no averaging is performed before filtering. It is the case that uses the maximum number of instantaneous CFR estimates.

It can be seen that the performance of random pilot pattern method is worse than that of fixed pattern while the computational complexity of the random pilot pattern method is much higher than that of fixed pattern method. It can be explained by the fact that in the fixed pattern case, the instantaneous CFR estimates are averaged before interpolation, and the accuracy of CFR estimates on virtual pilot positions significantly affects the MSE of overall CFR after Wiener filtering. The rectangular shape pilot pattern has almost the same performance as diamond shape pattern. Hence the rectan- 


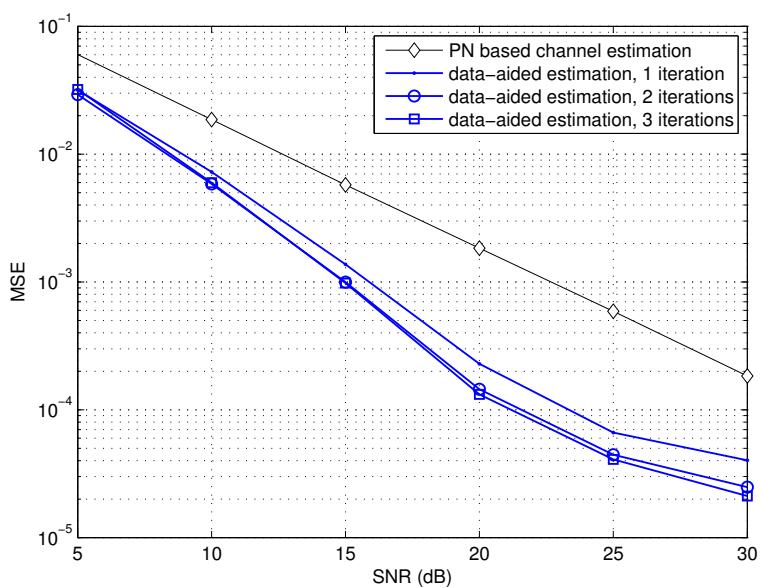

Fig. 5. MSE performance of the proposed data-aided channel estimation method with 16QAM in TU-6 channel with velocity of $30 \mathrm{~km} / \mathrm{h}$. Rectangular shape pilot pattern with $L_{t}=4, L_{f}=9$. Interpolation block length $B=21$.

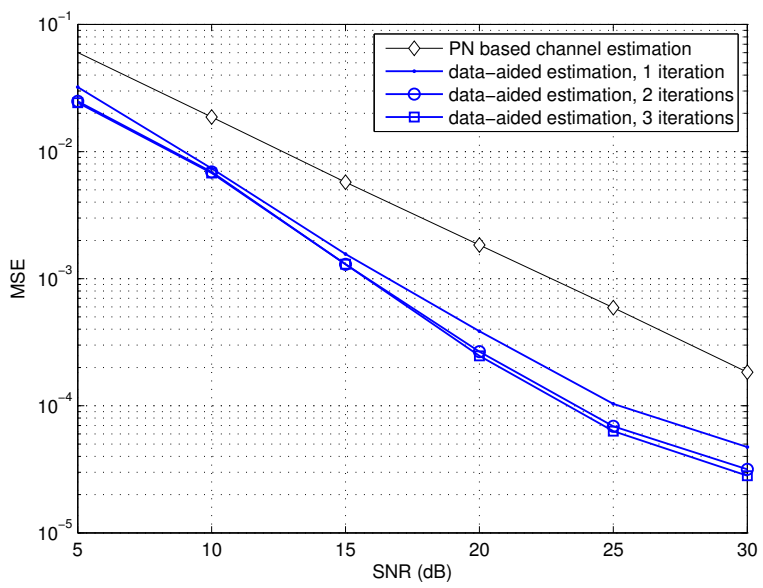

Fig. 6. MSE performance of the proposed data-aided channel estimation method with 64QAM in TU-6 channel with velocity of $30 \mathrm{~km} / \mathrm{h}$. Rectangular shape pilot pattern with $L_{t}=4, L_{f}=9$. Interpolation block length $B=21$.

gular shape pattern is used in the following simulations for the sake of implementation simplicity. When signal-to-noise ratio (SNR) is low, since the rebuilt data symbols are not reliable, performing average in larger averaging region provides better performance, e.g. the cases of $L_{t}=8, L_{f}=9$ are better than $L_{t}=4, L_{f}=9$. On the contrary, the rebuilt data symbols are quite accurate when SNR is high. Thus, small average region can sufficiently reduce the estimation error, while smaller pilot spacing provides better interpolation results. For instance, the performance floor, which mainly comes from the interpolation error and the channel variation within the coherence region, is lower in $L_{t}=4, L_{f}=9$ case than that in $L_{t}=8, L_{f}=9$ case. Interestingly, as no averaging over several consecutive OFDM symbols is performed in the random pilot shape case, the channel variation does not introduce estimation error. Therefore, there is no performance floor at high SNR with the random pilot shape.

Fig. 5 and 6 give the MSE performance of the proposed method in TU-6 channel with a velocity of $30 \mathrm{~km} / \mathrm{h}$ with

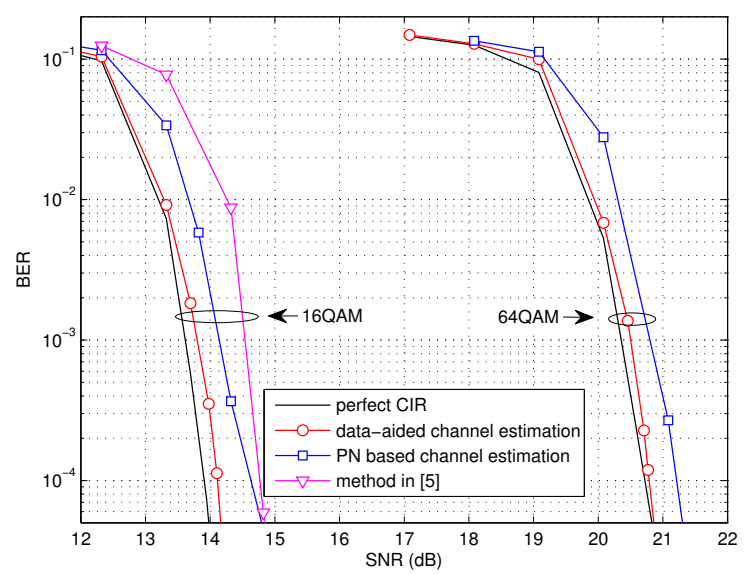

Fig. 7. BER of the DTMB system using the proposed data-aided channel estimation for 16QAM and 64QAM after 2 iterations in TU-6 channel with velocity of $30 \mathrm{~km} / \mathrm{h}$. Rectangular shape pilot pattern with $L_{t}=4, L_{f}=9$. Interpolation block length $B=21$. LDPC code rate 0.6.

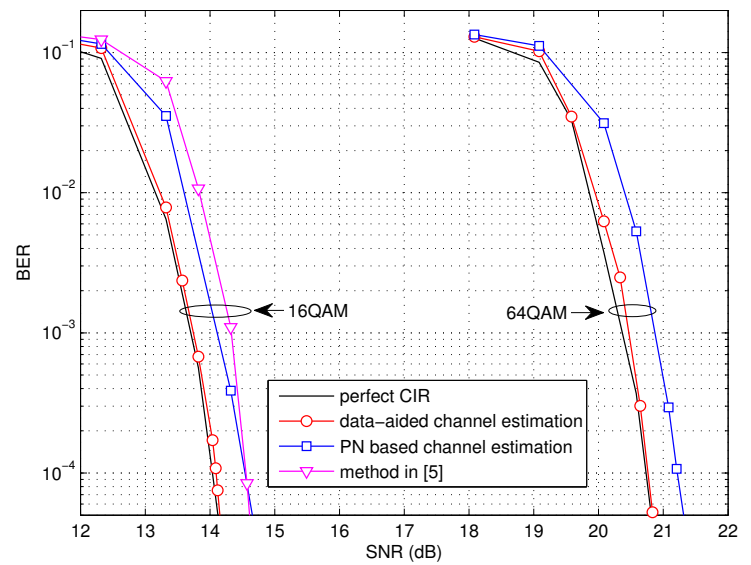

Fig. 8. BER of the DTMB system using the proposed data-aided channel estimation for 16QAM and 64QAM after 2 iterations in TU-6 channel with velocity of $120 \mathrm{~km} / \mathrm{h}$. Rectangular shape pilot pattern with $L_{t}=2, L_{f}=9$. Interpolation block length $B=21$. LDPC code rate 0.6.

16QAM and 64QAM, respectively. For both cases, the proposed method provides satisfactory performance after only two iterations. The performance with iterations more than three converges. Therefore, the iteration time is set to two in the BER performance evaluation in this paper. Not surprisingly, the proposed method performs better with 16QAM given the same SNR, because the rebuilt data symbols are more reliable in this case. The proposed method provides $7.8 \mathrm{~dB}$ gain in terms of required SNR to achieve MSE of $1 \times 10^{-3}$ over PN based channel estimation method with 16QAM. From Fig.6, the gain is accordingly $6.9 \mathrm{~dB}$ with 64QAM given MSE of $1 \times 10^{-3}$. As higher order modulation normally requires more accurate channel estimation, the gain is also evaluated with a lower MSE level, say $2 \times 10^{-4}$. The gain over PN based channel estimation increases to $8.7 \mathrm{~dB}$.

Fig. 7 and 8 present the bit error rate (BER) of the DTMB system using the proposed method with velocities of $30 \mathrm{~km} / \mathrm{h}$ and $120 \mathrm{~km} / \mathrm{h}$, respectively. Interestingly, performance using 
the proposed method is very close to that using perfect channel estimation. Only 0.1 to $0.2 \mathrm{~dB}$ gaps can be found. The proposed method provides more than $0.5 \mathrm{~dB}$ gain over the PN based estimation method in terms of required SNR to achieve a BER of $5 \times 10^{-5}$. Considering that the LDPC code with code rate 0.6 is used in the simulation, this improvement is quite significant. Even more gain can be expected if a weaker channel coding is adopted. The latest channel estimation method dedicated to TDS-OFDM proposed in [5] is also simulated for comparison. It needs a computational complexity equivalent to the proposed method. As [5] does not give the partial decision boundary for 64QAM, for the fairness of the comparison, only the performance for 16QAM is presented. The results are obtained with three iterations in the channel estimation. It can be found that the proposed method outperforms the method in [5] $0.7 \mathrm{~dB}$ and $0.5 \mathrm{~dB}$ with velocities of $30 \mathrm{~km} / \mathrm{h}$ and $120 \mathrm{~km} / \mathrm{h}$, respectively.

\section{CONCLUSION}

This paper proposes an iterative data-aided channel estimation algorithm with reduced complexity. In contrast to turbo channel estimation, the proposed algorithm does not include the decoding and interleaving functions when rebuilding the data symbols. A new joint 2-D channel estimate refinement and interpolation is proposed to provide efficient estimation performance. Moreover, the proposed algorithm is adaptable to the non-uniform-power modulation cases as exemplified by the given 16QAM and 64QAM results. Interestingly, the BER performance obtained when applying our method to the DTMB system is not only superior to that using the classical PN based method and the latest method in literature [5], but also close to that with perfect channel estimation, which demonstrates the efficiency of the algorithm.

\section{REFERENCES}

[1] S. Tang, F. Yang, et. al. "Iterative channel estimation for block transmission with known symbol padding - a new look at TDS-OFDM," in Proc. of the IEEE GLOBECOM'07, Nov. 2007, pp. 4269-4273.

[2] Framing Structure, Channel Coding and Modulation for Digital Television Terrestrial Broadcasting System, Chinese Standard GB20600-2006.

[3] H. Steendam and M. Moeneclaey, "Different guard interval techniques for OFDM: performance comparison," in Proc. of MC-SS'07, Herrsching, Germany, May 2007.

[4] M. Muck, M. de Courville, M. Debbah and P. Duhamel, "A pseudo random postfix OFDM modulator and inherent channel estimation techniques", Proc. IEEE Global Telecommunications Conference (Globecom'03), San Francisco, USA, vol.4, pp. 2380-2384, Dec. 2003.

[5] S. Tang, K. Peng, et. al. "Novel decision-aided channel estimation for TDS-OFDM systems," in Proc. of IEEE ICC'08, Beijing, China, May 2008, pp. 946-950.

[6] M. Zhao, Z. Shi and M. C. Reed, "Iterative turbo channel estimation for OFDM system over rapid dispersive fading channel," IEEE Trans. Wireless Commun., vol. 7, no. 8, pp. 3174-3184, Aug. 2008.

[7] M. Liu, M. Crussiere and J.-F. Helard, "A combined time and frequency algorithm for improved channel estimation in TDS-OFDM," in Proc. of IEEE ICC'10, Cape Town, South Africa, May 2010.

[8] Y. Li, "Pilot-symbol-aided channel estimation for OFDM in wireless systems," IEEE Trans. Veh. Technol., vol. 49, pp. 1207-1215, Aug. 2002.

[9] F. Tosato and P. Bisaglia, "Simplified soft-output demapper for binary interleaved COFDM with application to HIPERLAN/2," in Proc. of IEEE ICC'02, New York City, April 2002, pp. 664-668.

[10] P. Hoeher, S. Kaiser, and P. Robertson, "Pilot-symbol-aided channel estimation in time and frequency," in Proc. IEEE GLOBECOM'97, Phoenix, USA, Nov. 1997, pp. 90-96.

[11] X. Dong, W.-S. Lu and A.C.K. Soong, "Linear interpolation in pilot symbol assisted channel estimation for OFDM," IEEE Trans. on Wireless Commun., vol. 6, no. 5, pp. 1910-1920, May 2007.

[12] J.-W. Choi, Y.-H. Lee, "Optimum pilot pattern for channel estimation in OFDM systems," IEEE Trans. on Wireless Commun., vol. 4, no. 5, pp. 2083-2088, Sept. 2005.

[13] T. Rappaport, Wireless Communications: Principles and Practice, 2nd Edition. Prentice-Hall, 2001. 